unter dem zunehmenden ökonomischen Druck bis 2013. Die wegen fehlender Übergangsvorschriften sehr ungerechte Unterhaltsrechtsreform dagegen wird bleiben und ist Sache von Anwältinnen, Anwälten und Gerichten. Die betroffenen Frauen bieten kein ökonomisches Potenzial, das zu fördern sich lohnen würde.
Das Verfassungsgericht: Berlin oder Karlsruhe! Sie haben die Wahl!

Ich wünsche uns einen schönen Abend mit anregenden Gesprächen und bedanke mich für Ihre Aufmerksamkeit.

\title{
Rezension: Marianne Weber. Leben und Werk
}

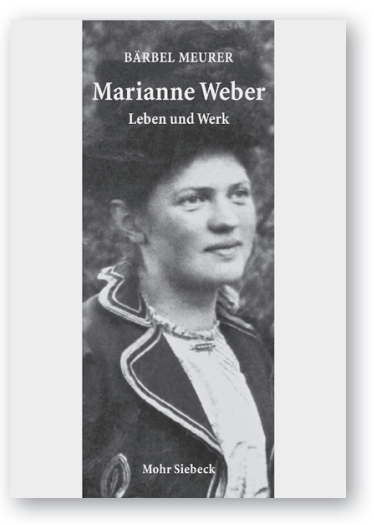

\author{
Bärbel Meurer \\ Marianne Weber. Leben und Werk \\ Mohr Siebeck Verlag, Tübingen \\ 2010 \\ ISBN-10 3161504526 \\ ISBN-13 9783161504525 \\ Gebunden, 688 Seiten, 89,00 EUR
}

Bärbel Meurer, die bereits 2004 den ersten Sammelband herausgegeben hat, der die Person Mariannes - nicht Max Webers - in den Mittelpunkt des Forschungsinteresses gestellt hat, legt nun im Anschluss eine dringend erwünschte erste Biografie Marianne Webers vor. Dass diese Biografie im Mohr Siebeck Verlag erschienen ist, dem die Webers bereits zu Lebzeiten eng verbunden waren, ist ein schönes Detail. Meurer selbst geht davon aus, dass ihr „Buch über Marianne Weber für lange Zeit die einzige Monografie und Biografie bleiben wird“. Vor dem Hintergrund, dass in den letzten Jahren viele biografische Arbeiten über Führungspersönlichkeiten der deutschen Frauenbewegungen veröffentlicht worden sind und einige Arbeiten mehr am Entstehen sind, bleibt zu hoffen, dass dies nicht der Fall sein wird, sondern durch die Biografie im Gegenteil neue Arbeiten zu Marianne Weber angeregt werden.

Die Arbeit ist einerseits so gegliedert, dass es acht große Kapitel gibt, die wiederum in Unterkapitel unterteilt und im Index so aufgeführt sind, dass die Leserin oder der Leser gut wählen kann, welche der Kapitel sie oder ihn interessieren und welche sie oder er vielleicht lieber überspringen mag. Die Autorin hat damit quasi Leben und Werk voneinander zu trennen versucht, was einerseits einen gezielten Zugriff ermöglicht, andererseits aber auch zu unnötigen und teils wörtlichen Wiederholungen führt.

Das Buch führt chronologisch in das Leben Marianne Webers ein, über ihren Familienhintergrund, den sie teils mit Max Weber teilte, über ihre unglückliche Kindheit, ihren Zeichenunterricht in Berlin - wo sie Max Weber kennen lernte über die verschiedenen Phasen und Reisen des Ehepaares Weber, dem Werk Marianne Webers sowie ihrem Engagement in der Frauenbewegung als auch in der Politik bis zum Tode Max Webers. Sie beschreibt die Mammutaufgabe der Witwe, die von Familie, Freundinnen und Freunden, Kolleginnen und Kollegen und Bekannten die Briefe des Verstorbenen erbeten hatte, alle hinterlassenen Manuskripte gesichtet, geordnet und größtenteils ediert hat sowie jenes „Lebensbild“ Max Webers geschrieben hat, das bis heute die Grundlage der gesamten biografisch orientierten Weber-Forschung ist. Auch die Zeit des Nationalsozialismus, Marianne Webers Haltung zu dem Regime sowie das Alter mit den Kontakten zur Kögener Bewegung sowie zur Odenwaldschule, an die alle ihre Adoptivkinder gingen, kommen zur Sprache.

Die Autorin hat die Absicht, die Figur Marianne Webers, die wissenschaftlich selbstständig neben ihrem Mann tätig war, aus dem Schatten Max Webers heraustreten zu lassen. Der Versuch, in Abgrenzung zu seiner wissenschaftlichen Arbeit die gegenseitigen Einflüsse des Ehepaares auf ihr jeweiliges Schaffen auszuloten, führt allerdings dazu, dass die Biografie Marianne Webers sich am Ende genauso ausführlich und gelegentlich langatmig mit dem Arbeitsleben und den Plänen Max Webers beschäftigt wie mit denen Mariannes. Auf diese Art entsteht mehr ein sehr lesbares und zeitweise amüsantes Doppelporträt der Webers als eine Biografie Mariannes. Trotzdem lässt sich aus dem Buch naturgemäß mehr über Marianne Weber erfahren, als bisher bekannt war. Leider aber auch deutlich weniger, als die Leserin und der Leser einer Biografie Marianne Webers gerne erfahren würde.

Das beginnt bereits damit, dass das Vorhaben Meurers, den gegenseitigen Einfluss der Webers aufeinander zu erforschen, etwas diffus bleibt. Dass ein Ehepaar, das eng zusammenlebt und -arbeitet, gegenseitigen Einfluss aufeinander ausgeübt haben mag, ist zu vermuten und beweist sich alleine durch die Editionsmeisterleistung Mariannes nach dem Tod von Max. Ohne ein umfassendes Verständnis für die Arbeit ihres Mannes, das eine jahrelange Teilnahme daran voraussetzt, hätte sie diese Aufgabe nicht leisten können. Ob Max Weber wiederum seine Rationalismus-Idee von Marianne übernommen hat oder inwieweit er direkt oder indirekt Einfluss auf ihre Arbeit genommen hat, scheint mir am Ende vielleicht auch weniger interessant als die Frage, inwiefern Marianne Webers Werk mit oder ohne Einfluss ihres Mannes einen selbstständigen Wert hat. Obwohl die Autorin das Werk Mariannes beschreibt, versäumt sie, es in den zeitgenössischen wissenschaftlichen Hintergrund einzuordnen. So bleibt trotz der Einschätzung des Werkes „Ehefrau und Mutter in der 
Rechtsentwicklung“, die im Vergleich zu dem Sammelband von 2004 nichts Neues bringt, am Ende unklar, worin der große Einfluss Marianne Webers gelegen hat. Dies gilt ebenso für den Beitrag Marianne Webers zur Debatte innerhalb der Frauenbewegung; es wird beispielsweise nicht deutlich, worin im Gegensatz zu den Forderungen zum Eherecht von Emilie Kempin, Marie Raschke und Selma Proel $\beta$ die große Bedeutung in Marianne Webers Beitrag zum Eherecht gelegen haben soll. Dies ist vor allem deshalb zu bedauern, da tatsächlich - wie die Autorin ja auch behauptet - das Werk ein Novum sowohl für die Rechtswissenschaft als auch im Kontext der Eherechtsforderungen der Frauenbewegung darstellte.

Hier ist schon angedeutet, dass ein großer Schwachpunkt der Arbeit ist, dass sie überwiegend deskriptiv und ohne wissenschaftliche Tiefe bleibt. Das liegt zum einen an der Quellenbasis, die die Autorin nutzt, um das Leben Marianne Webers in den Griff zu bekommen. Statt auf eine Vielfalt von möglichen Quellen zuzugreifen, schildert sie das Leben der Webers überwiegend aus den Briefen Mariannes an ihre Schwiegermutter. Sind die vielen zitierten Einschübe ihrer Briefe zwar originell sowie sehr interessant und machen das Buch lebendig, kann man sich manchmal nicht des Eindrucks erwehren, dass man möglicherweise lieber gleich eine editierte Briefsammlung lesen würde. Gleichzeitig werden Ereignisse geschildert, ohne dass sie mit Sekundärliteratur und historischem Wissen des Zeitgeschehens verifiziert oder gar in den Gesamtzusammenhang eingeordnet werden. Daraus ergeben sich zuweilen nicht nur sachliche Fehler, sondern auch Momente der Irritation, weil man zu den in dem Brief geschilderten Ereignis nicht nur gerne mehr gewusst hätte, sondern dazu auch bereits Sekundärliteratur existiert, die diese Lücke hätten füllen können und müssen. Es ist auffallend oft der Fall, dass die Autorin Sekundärliteratur zu Phasen und Ereignissen des Lebens der Webers nicht gelesen oder zumindest nicht verarbeitet hat. Sie erwähnt zum Beispiel den Besuch Marianne Webers bei Jane Addams in Chicago ohne einen Bezug zu deren wichtigen Tätigkeiten und einen Literaturhinweis. Genauso wenig Beachtung findet die Frage der Gleichberechtigung in der Weimarer Verfassung oder die Behandlung der Unehelichenfrage in der Nationalversammlung, obwohl diese für die Frauenbewegung und Marianne Weber von ungeheurer Bedeutung sind. Sekundärliteratur wird vor allem nur dann genutzt, wenn die Autorin bestehende Urteile - zum Beispiel bezüglich der Ehe der Webers und den tatsächlichen oder vermeintlichen Geliebten Max Webers, Mina Tobler und Else von Jaffé, ob berechtigt oder nicht sei dahingestellt - widerlegen will. In den Phasen, die das Eheleben bzw. das außereheliche Leben Max Webers betreffen, wird auch eine der von der Geschichtswissenschaft häufig angesprochenen Gefahren des Biografieschreibens deutlich: Biografinnen und Biografen, die sich in die Person ihrer Biografie vernarren, neigen dazu, ein leicht verzerrtes Bild von ihr zu zeichnen, indem sie die Fakten in das Bild pressen, das sie sich von der Person gemacht haben.
Desiderate - und das gibt die Autorin in ihrem Vorwort selbst $\mathrm{zu}$ - finden sich vor allem in Bezug auf die Frauenbewegung, der - neben der Werkherausgabe des Gesamtwerkes Max Webers - zentralen Lebensaufgabe von Marianne Weber. Hier hätte man sich eine andere Schwerpunktsetzung der Autorin gewünscht, denn gerade dieser Aspekt im Leben Marianne Webers hätte viele interessante neue Erkenntnisse bringen können. Das Archiv des Bundes deutscher Frauenvereine (BDF), das die Autorin nicht vollständig ausgewertet hat, ist tatsächlich nicht so umfassend, als dass man ein Zögern hier verstehen würde. Gerade auch im Gebiet von Marianne Webers Spezialistentum, den Rechtsforderungen der Frauenbewegung im Familienrecht, hätte die Autorin ein weites Feld der Betätigung gehabt, das sie nicht genutzt hat. So bleibt zum Beispiel die von Marianne Weber 1919 neu eingesetzte Rechtskommission ebenso unerwähnt wie die Rechtsarbeit des BDF in diesen Jahren. Auch hier leidet die Arbeit immer wieder darunter, dass die in den Briefen erwähnten Ereignisse nicht kontextiert werden.

Interessant und überzeugend ist die Autorin allerdings in ihrer ablehnenden Haltung zu dem bisherigen Trend in der Forschung, dass mit dem Übergang des BDF-Vorsitzes von Marie Stritt an Gertrud Bäumer eine erste „Rechtswende“ und ein „Richtungswechsel“ des Vereins und damit eine Schwächung der Frauenbewegung verbunden gewesen sei. Sie arbeitet zu Recht heraus, dass das gesamte Personal des engeren Vorstands von 1900 bis zum Ende der Weimarer Republik fast gleich geblieben sei und Marie Stritt nicht in einer „Palastrevolution “ abgesetzt worden war, sondern in einem demokratischen Wahlverfahren abgewählt wurde. Tatsächlich war ihre Person schon lange wegen ihres Führungsstils in die Kritik geraten. Auch überzeugt die Wertung dahingehend, dass die Person der Vorsitzenden langfristig für die Politik des Bundes meistens keine ausschlaggebende Rolle in den großen Entscheidungen gespielt habe, was nicht zuletzt in den Jahren von Marianne Webers Vorsitz des BDF deutlich wurde. Genauso betont sie völlig zu Recht, dass der BDF in den Jahren der Weimarer Republik keineswegs so passiv war, wie das in der bisherigen Literatur dargestellt wird. Auch die Person Gertrud Bäumers und ihr Einfluss auf den BDF erfährt eine Korrektur dahingehend, dass sie möglicherweise keine so beherrschende Figur im BDF war, wie bisher dargestellt wurde, sondern im Gegenteil eine große Kontinuität in der Führung des BDF von Marie Stritt bis Emma Ender bestanden hat. Auch zu der Haltung des BDF zur Aufnahme rechtsstehender, konservativer Verbände finden sich klare Worte, die der seit Richard Evans Buch über die deutsche Frauenbewegung bestehenden These von dem schleichenden Konservatismus des BDF bis zu einem Aufgeben im Nationalsozialismus widerspricht.

Alles in allem ist die Biografie aber wirklich unterhaltsam und lesenswert, wenn sie auch deutlich mehr hätte leisten können und sollen. (Marion Röwekamp: Dr. Marion Röwekamp, Feodor Lynen Fellow der Alexander von Humboldt Stiftung am Colmex, CIESAS und der UNAM, Mexico City) 